\title{
Anti-resonance features of destructive quantum interference in single-molecule thiophene junctions achieved by electrochemical gating
}

Jie Bai ${ }^{1+}$, Abdalghani Daaoub ${ }^{2+}$, Sara Sangtarash ${ }^{2+}$, Xiaohui $\mathrm{Li}^{1+}$, Yongxiang Tang ${ }^{1}$, Qi Zou ${ }^{3}$, Hatef Sadeghi ${ }^{2}$, Shuai Liu ${ }^{1}$, Xiaojuan Huang ${ }^{1}$, Zhibing Tan ${ }^{1}$, Junyang Liu ${ }^{1}$, Yang Yang ${ }^{1}$, Jia Shi ${ }^{1}$, Gábor Mészáros $^{4}$, Wenbo Chen $^{3 *}$, Colin Lambert ${ }^{2 *}$, Wenjing Hong ${ }^{1 *}$

${ }^{1}$ State Key Laboratory of Physical Chemistry of Solid Surfaces, iChEM, College of Chemistry and Chemical Engineering \& Pen-Tung Sah Institute of Micro-Nano Science and Technology, Xiamen University, Xiamen 361005, China

${ }^{2}$ Department of Physics, Lancaster University, Lancaster LA1 4YB, United Kingdom

${ }^{3}$ Shanghai Key Laboratory of Materials Protection and Advanced Materials in Electric Power, Shanghai University of Electric Power, Shanghai 200090, China

${ }^{4}$ Institute of Materials and Environmental Chemistry, Chemical Research Centre, Hungarian Academy of Sciences, Pusztaszeri út 59-67, H-1025 Budapest, Hungary

*Email:whong@xmu.edu.cn; c.lambert@lancaster.ac.uk; wenbochen@shiep.edu.cn

${ }^{+}$These authors contributed equally to this work.

Controlling the electrical conductance and in particular the occurrence of quantum interference in single-molecule junctions through gating effects, has potential for the realization of highperformance functional molecular devices. In this work, we used an electrochemically-gated, mechanically-controllable break junction technique to tune the electronic behaviour of thiophene-based molecular junctions that show destructive quantum interference (DQI) features. By varying the voltage applied to the electrochemical gate at room temperature, we 
reached a conductance minimum that provides direct evidence of charge transport controlled by an anti-resonance arising from DQI. Our molecular system enables conductance tuning close to two orders of magnitude within the non-faradaic potential region, which is significantly higher than that achieved with molecules not showing DQI. Our experimental results, interpreted using quantum transport theory, demonstrate that electrochemical gating is a promising strategy for obtaining improved in-situ control over the electrical performance of interference-based molecular devices.

The understanding of charge transport through molecular building blocks is essential for the design of functional electric materials and devices from the molecular level. Charge transport at the nanoscale is dominated by quantum effects that can be controlled by the electron wave function. Among these effects, destructive quantum interference (DQI) in single-molecule junctions describes a quantum process in which the electron waves propagate through separated molecular orbitals and combine destructively ${ }^{1-4}$. This effect will lead to a reduction in the electron transmission probability and therefore a suppression of molecular conductance by orders of magnitude compared with molecular junctions without $\mathrm{DQI}^{1,3}$. Consequently, the control of DQI in single-molecule junctions offers a promising strategy for developing high-performance molecular devices such as molecular switches ${ }^{5}$, transistors $^{6}$ and thermoelectric devices ${ }^{7}$. In previous works, efforts for controlling DQI utilized chemical design to manipulate the molecular architectures, including electronic structure modification $^{8}$, molecular topologies ${ }^{9}$, heteroatom substitution ${ }^{10}$, and even chemical reactions to change the molecular structures ${ }^{11}$. However, such indirect methods do not allow flexible and in-situ control and the fine tuning of interference still remains a major challenge for the fabrication of interference based molecular devices, such as single-molecule transistors.

Although electrostatic gating offers an integrative approach for the tuning of the relative positions of molecule orbitals at different temperatures ${ }^{12,13}$, electrochemical gating provides a complementary 
and effective approach to manipulate charge transport in the electrochemically active or inert molecular junctions at room temperature ${ }^{14-17}$. When the applied electrode potentials vary in a nonfaradaic region, the molecular energy alignment relative to the electrode Fermi energy would be tuned $^{18}$, which offers the in-situ fine tuning of charge transport through single-molecule junctions. Since the quantum interference in the charge transport through single-molecule junctions is energy dependent, the continuous tuning of the electrode potential offers an ideal strategy for realizing interference-based single-molecule electrochemical transistors $(\mathrm{ECTs})^{16-22}$, and the high gating efficiency and relatively large gate voltage windows provide opportunities for gating single-molecule junctions between resonances associated with molecular energy levels and anti-resonances associated with DQI ${ }^{18,23}$.

Herein, we develop a mechanically controllable break junction technique (MCBJ) chip integrated with the electrochemical gate for the fabrication of single-molecule ECTs in ionic liquid, and investigate the charge transport through single-molecule thiophene junctions with DQI controlled by electrochemical gating. We observe the anti-resonance from DQI in a non-faradaic region at around $-0.4 \mathrm{~V}$ versus $\mathrm{Ag} / \mathrm{AgCl}$ quasi-reference electrode, which offers direct evidence of that charge transport is controlled by DQI. It is also found that the single-molecule thiophene junctions with DQI shows $\sim 100$ times conductance enhancement as the potential moves positively, which is significantly larger than that without DQI. Density functional theory (DFT) calculations of the electrochemical double layers around the molecular backbones, confirm that the gating shifts the DQI anti-resonance feature towards the Fermi energy.

The single-molecule conductance measurements were carried out on an electrochemical tip-bead (ECTB) chip integrated with four electrodes for electrochemical gating using MCBJ technique (Fig. 1a). The two working electrodes (source and drain electrodes) fabricated from a Au tip coated with insulating glue and a Au bead, are separated with a gap of $\sim 20 \mu \mathrm{m}$ and fixed on a stainless steel sheet 
using epoxy resin. A Pt wire and a $\mathrm{Ag}$ wire coated with a layer of $\mathrm{AgCl}$ are introduced as the counter and reference electrodes, respectively. This asymmetric architecture of the source and drain electrodes is inherited from STMBJ method, and the integrated electrochemical system on a MCBJ chip can be employed as the on-chip single-molecule ECTs. During the conductance measurements, the gap between two working electrodes was further decreased until connected through bending the chip downwards driven by stepper motor and piezo stacks, and then repeated breaking/connecting process (see Supplementary Information S2 for details).
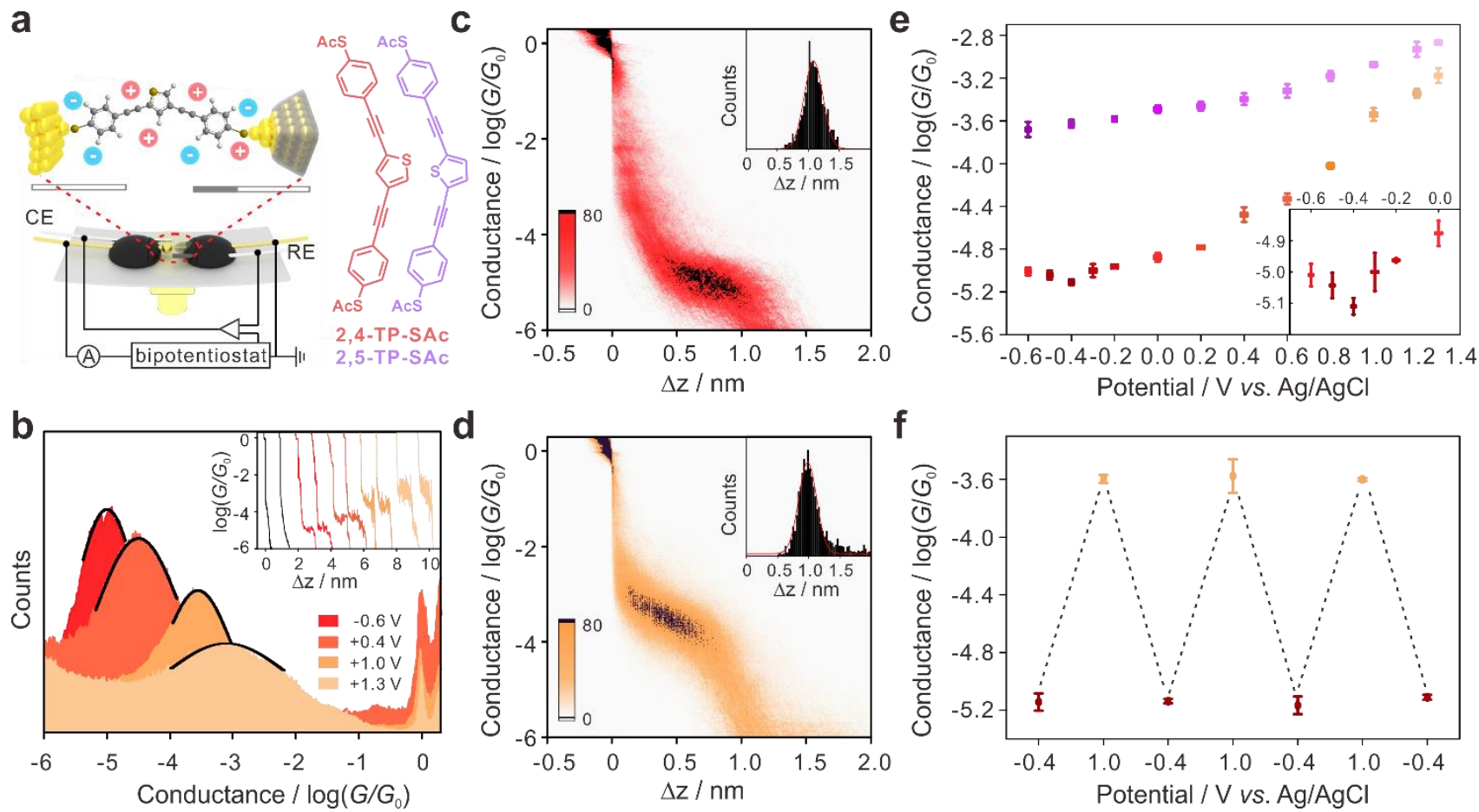

Fig. 1 | Electrochemical gating of 2,4-TP-SAc molecular junctions. a, Schematics of the electrochemically-gated, mechanically controllable break junction technique and molecular structures of thiophene derivatives with the anchoring groups of thioacetyl (-SAc). b, One-dimensional (1D) conductance histograms of 2,4-TP-SAc at different potentials, and the potentials of coated Au tip relative to $\mathrm{Ag} / \mathrm{AgCl}$ were chosen as the applied electrode potentials, while the potential of $\mathrm{Au}$ bead were set as $100 \mathrm{mV}$ lower to have the bias of $100 \mathrm{mV}$ between the two electrodes. Inset: typical individual traces of 2,4-TP-SAc at different potentials: black for pure solvent, red for 2,4-TP-SAc junctions at $-0.6 \mathrm{~V}$, orange for $0.4 \mathrm{~V}$, yellow for $1.0 \mathrm{~V}$ and light yellow for $1.3 \mathrm{~V}$. Two-dimensional 
(2D) conductance-displacement histograms of 2,4-TP-SAc at $-0.6 \mathrm{~V}(\mathbf{c})$ and $1.0 \mathrm{~V}(\mathbf{d})$, and the relative displacement distributions (insets). e, Tendency of the molecular conductance of 2,5-TP-SAc (purple) and 2,4-TP-SAc (orange) versus electrode potentials from $-0.6 \mathrm{~V}$ to $1.3 \mathrm{~V}$. Inset: magnification from $-0.2 \mathrm{~V}$ to $0.5 \mathrm{~V}$. f, Reversible switching of 2,4-TP-SAc under the sweep potentials between $-0.4 \mathrm{~V}$ and 1.0 V. The error bars of the conductance value were determined from the variation of the most probable conductance values in three independent conductance measurements, and the error bars of the potential were determined from the potential stability test (Supplementary Fig. 2a).

To reveal the role of DQI in the charge transport through gated single-molecule junction, we investigate the charge transport through the thiophene core molecules with (2,4-TP-SAc) and without (2,5-TP-SAc) DQI as shown in Fig. 1a. Fig. 1b (inset) shows several typical individual stretching traces for 2,4-TP-SAc with the electrode potentials at $-0.6 \mathrm{~V}, 0.4 \mathrm{~V}, 1.0 \mathrm{~V}$ and $1.3 \mathrm{~V}$ versus the reference potential. The black traces show a tunneling decay after the rupture of Au atomic contact in pure solvent, while molecular plateaus corresponding to the single-molecule conductance are found in the traces in solution with target molecules. The position of the molecular plateau shifts with the electrode potentials, suggesting the feasibility of electrochemical gating of single-molecule junctions using MCBJ technique. For further analysis, hundreds to thousands of such conductance traces were used to construct the conductance histograms. Fig. 1b shows the 1D conductance histograms of 2,4TP-SAc molecule measured at different potentials. A pronounced conductance peak located at $10^{-5.0 \pm 0.03} G_{0}$ (conductance quantum, $G_{0}=2 e^{2} / h$ ) was obtained for 2,4-TP-SAc at $-0.6 \mathrm{~V}$, and shifted for almost two orders of magnitude to $10^{-3.2 \pm 0.07} G_{0}$ at $1.3 \mathrm{~V}$. To exclude the conductance differences from configurational change, we constructed the 2D conductance-displacement histograms of 2,4TP-SAc at $-0.6 \mathrm{~V}$ and $1.0 \mathrm{~V}$, as shown in Fig. 1c and 1d. By accounting for the Au-Au snap back distance, the molecular length obtained from the relative stretched distance distribution is determined to be $\sim 1.5 \mathrm{~nm}$ (1.0 nm determined from the relative displacement distribution, and adding a $\mathrm{Au}-\mathrm{Au}$ 
snap back distance of $\sim 0.5 \mathrm{~nm}^{24}$ ). This is quite similar to the calculated molecular length, suggesting the conductance enhancement is ascribed to the applied electrode potentials rather than configurational change.

To reveal the variation of the molecular conductance with electrode potentials within whole nonfaradaic region, we measured the molecular conductance while varying the electrode potentials from $-0.6 \mathrm{~V}$ to $1.3 \mathrm{~V}$, which is limited by desorption of the molecules at lower potential and the Au oxidation at higher potential ${ }^{25}$ as shown in Fig. 1e (see Supplementary Fig. 2 for cyclic voltammetry measurements). When electrochemical gating was applied, the molecular conductance increases by $\sim 100$ times from $10^{-5.1 \pm 0.02} G_{0}$ at $-0.4 \mathrm{~V}$ to $10^{-3.2 \pm 0.07} G_{0}$ at $1.3 \mathrm{~V}$ without redox process. To further investigate the role of DQI in the electrochemical gated charge transport, we studied the conductance of 2,5-TP-SAc without DQI (see Supplementary Fig. 5 for details), which increases by only 8 times from $10^{-3.8 \pm 0.07} G_{0}$ at $-0.6 \mathrm{~V}$ to $10^{-2.9 \pm 0.02} G_{0}$ at $1.3 \mathrm{~V}$, indicating that 2,4-TP-SAc junctions with DQI provides more than one order of magnitude higher conductance tuning than that of the 2,5-TP-SAc junctions without DQI.

More importantly, it is found that the molecular conductance at $-0.6 \mathrm{~V}\left(10^{-5.0 \pm 0.03} G_{0}\right)$ is higher than that at $-0.4 \mathrm{~V}\left(10^{-5.1 \pm 0.02} G_{0}\right)$. To verify this tendency, we further carried out the experiments and plotted molecular conductance at $-0.3 \mathrm{~V}$ and $-0.5 \mathrm{~V}$ (Fig. 1e inset). Both conductance values at -0.3 $\mathrm{V}$ and $-0.5 \mathrm{~V}$ are slightly higher than that at $-0.4 \mathrm{~V}$, but lower than those at $-0.2 \mathrm{~V}$ and $-0.6 \mathrm{~V}$. The control experiments revealed that the conductance increase is not from the increase of faraday current (see Supplementary Fig. 7). The observation is in accordance with the anti-resonance tendency of DQI with the minimum conductance at around $-0.4 \mathrm{~V}$. This is direct evidence of the tuning of the antiresonance of DQI. Previous work has suggested that the transmission functions of molecular junction could be mapped electrochemically ${ }^{23}$. Herein we further demonstrate that the DQI can be directly mapped through electrochemical gating at room temperature. 
The high gating efficiency of 2,4-TP-SAc lead to the fabrication of the high-performance singlemolecule ECTs. Fig. 1f shows the reversible switching cycles of 2,4-TP-SAc under the sweep potentials between $-0.4 \mathrm{~V}$ and $1.0 \mathrm{~V}$. It is found that the conductance changes with the potential accordingly without any attenuation in conductance ratio. The large variation of the conductance provides a promising way to construct the molecular switch based on DQI, which tunes the electrode Fermi energy between the near resonance state and the anti-resonance state.

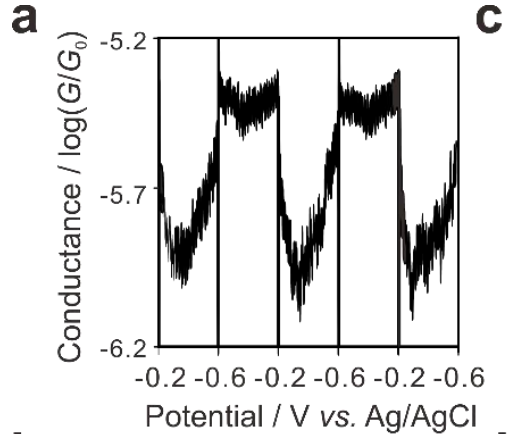

b

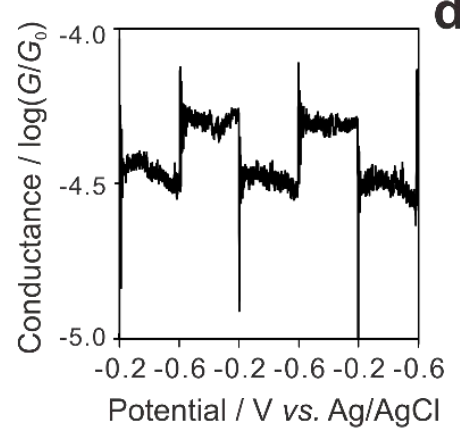

d
C
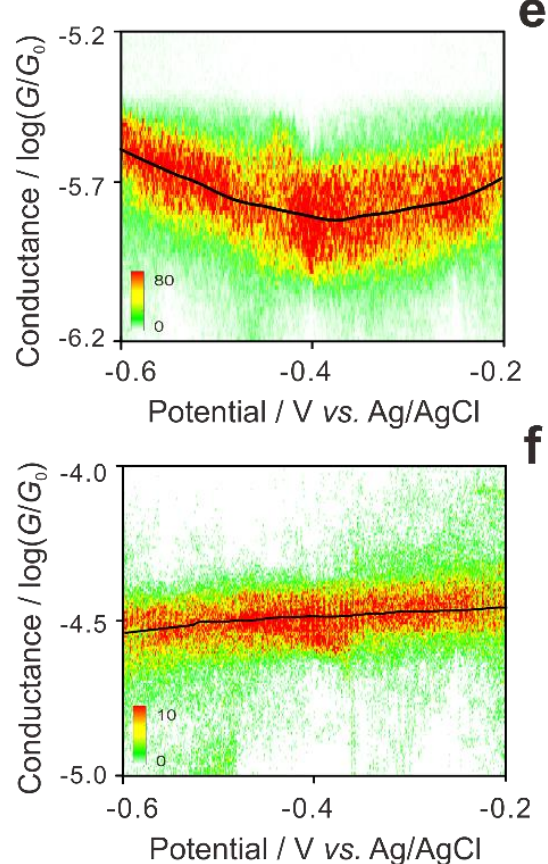

e

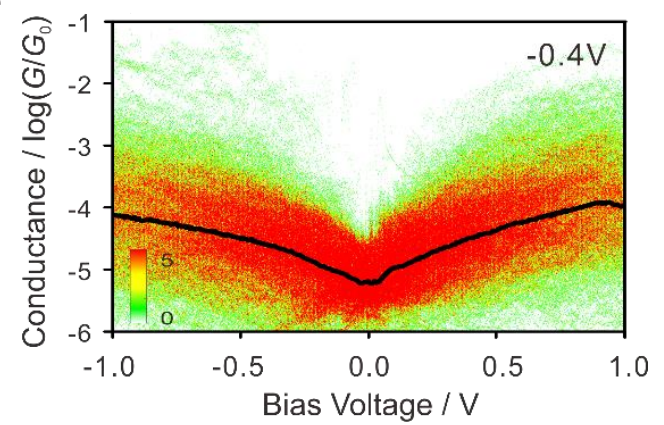

f

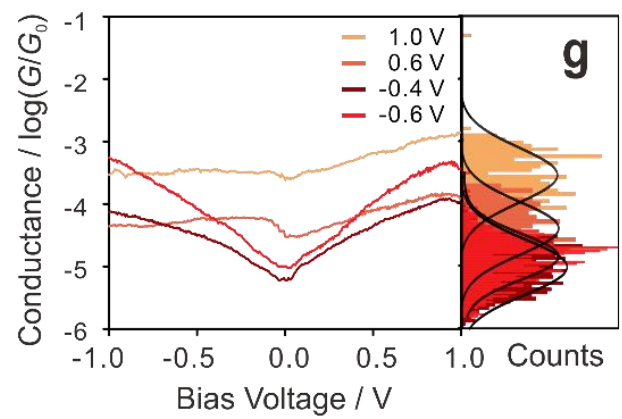

Fig. 2 | Conductance-electrode potential and current/conductance-voltage measurements of 2,4-

TP-SAc and 2,5-TP-SAc molecular junctions. a, Typical individual conductance-electrode potential traces of 2,4-TP-SAc. b, Typical individual conductance-electrode potential traces of 2,5-TP-SAc. c, 2D histogram of conductance-electrode potential of 2,4-TP-SAc (503 traces). d, 2D histogram of conductance-electrode potential of 2,5-TP-SAc (184 traces). e, 2D histogram and the most probable curve of current/conductance-voltage of 2,4-TP-SAc at -0.4 V (1154 traces). f, Most probable traces from 2D histogram of current/conductance-voltage of 2,4-TP-SAc at $1.0 \mathrm{~V}, 0.6 \mathrm{~V},-0.4 \mathrm{~V}$ and $-0.6 \mathrm{~V}$.

g, Conductance distributions of low-bias conductance determined from the slopes from $-100 \mathrm{mV}$ to $100 \mathrm{mV}$ in current/conductance-voltage traces and the count numbers are scaled for better comparison.

To further confirm the potential dependent conductance variation, we carried out the conductance- 
potential measurement for 2,4-TP-SAc and 2,5-TP-SAc in the same environmental conditions with the electrochemically-gated conductance measurement (see Methods for more details). As shown in Fig. $2 \mathrm{a}$ and $2 \mathrm{~b}$, there is a clear drop at around $-0.4 \mathrm{~V}$ in most of the individual conductance-electrode potential traces from the measurement of 2,4-TP-SAc, while most of the traces of 2,5-TP-SAc shows the increases with gate voltage from $-0.6 \mathrm{~V}$ to $-0.2 \mathrm{~V}$. It is found that the current determined from the conductance-electrode potential measurement is slightly lower than that from the electrochemicallygated conductance measurement, which may come from the preferred fully-stretched molecular configuration $^{24}$ as well as the contribution from faraday discharging current. Notably, the conductance drop in individual traces is found to be around half an order of magnitude, which is even higher than that determined from the electrochemically-gated conductance measurement. All data plots shown in Fig. 2c demonstrated the presence of a clear "V" shape node with a minimum at around $-0.38 \mathrm{~V}$ for 2,4-TP-SAc, which agrees well with the electrochemically gated conductance measurement and offers the direct evidence of anti-resonance from DQI. In contrast, the conductance-electrochemical potential traces of 2,5-TP-SAc show the slight increase shown in Fig. 2d.

We further carried out the current/conductance-voltage measurement at different potentials by sweeping the bias between $-1.0 \mathrm{~V}$ and $+1.0 \mathrm{~V}$ continuously at a sweeping rate of $5 \mathrm{~V} / \mathrm{s}^{24,26,27}$. Hundreds of current/conductance-voltage traces with molecular junctions are plotted in a two dimensional conductance-gate voltage histogram, and the typical conductance-gate voltage traces at $-0.4 \mathrm{~V}$ are shown in Fig. 2e, which exhibited a significant voltage dependence. We further compare the most probable current/conductance-voltage traces at different potentials. It is found that the current/conductance-voltage curves remained almost constant at $1.0 \mathrm{~V}$ for molecules, suggesting that the Fermi level are far from resonance or anti-resonance. More interestingly, it is found that the current/conductance-gate voltage at $-0.6 \mathrm{~V}$ is even more tilted than that of at $-0.4 \mathrm{~V}$. We further analyze the conductance distributions from current/conductance-voltage traces from the slopes from 
$-100 \mathrm{mV}$ to $100 \mathrm{mV}$. The most probable conductance from the slopes of current/conductance-voltage traces are determined to be $10^{-4.9 \pm 0.4} G_{0}$ at $-0.6 \mathrm{~V}, 10^{-5.0 \pm 0.5} G_{0}$ at $-0.4 \mathrm{~V}, 10^{-4.4 \pm 0.5} G_{0}$ at $0.6 \mathrm{~V}$, and $10^{-3.5 \pm 0.5} G_{0}$ at $1.0 \mathrm{~V}$ for 2,4-TP-SAc, which agrees well with the conductance measurement at fixed bias voltage (See supplementary Fig. 8 for the measurement of 2,5-TP-SAc).

a

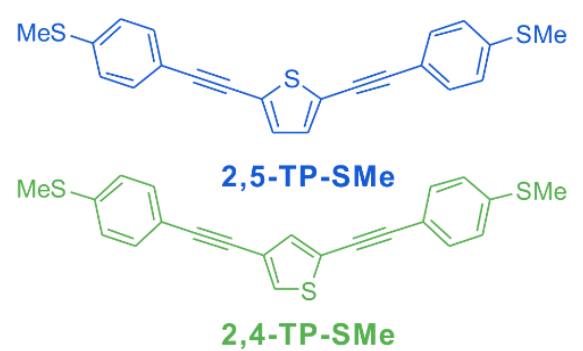

b

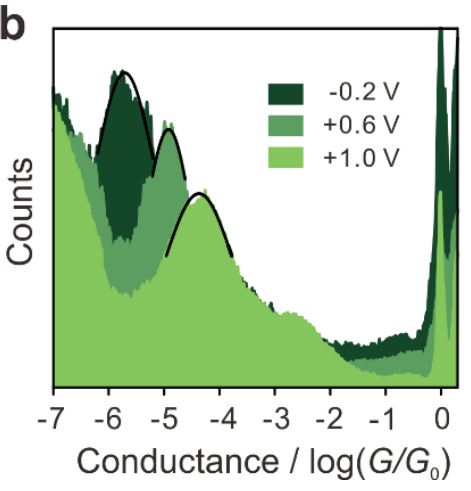

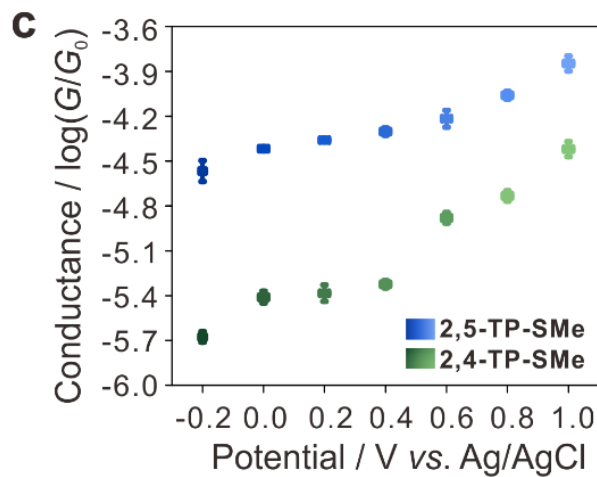

Fig. 3 | Transport characteristics of 2,4-TP-SMe and 2,5-TP-SMe molecular junctions. a, Molecular structures of thiophene derivatives with the anchoring group of thiomethyl (-SMe). b, 1D conductance histograms of 2,4-TP-SMe at different electrode potentials. c, Tendency of the molecular conductance of 2,4-TP-SMe and 2,5-TP-SMe versus electrode potentials from -0.2 to $1.0 \mathrm{~V}$. The error bars of the conductance value are determined from the variation of the most probable conductance values in three independent conductance measurements, and the error bars of the potential are determined from the potential stability test (Supplementary Fig. 2a).

To further verify the generality of this strategy and to study the role of electrode-molecule coupling, we replaced the anchoring group -SAc with -SMe and investigated the single-molecule conductance under electrochemical gating as shown in Fig. 3. It is found that the molecular conductance of 2,4-TPSMe increased by $\sim 20$ times from $-0.2 \mathrm{~V}$ to $1.0 \mathrm{~V}$, compared with $\sim 4$ times increase for 2,5-TP-SMe (see Supplementary Fig. 8 for details). This tendency is similar to that of 2,4-TP-SAc, except that the -SMe will desorb from Au when the electrode potential is negative than $-0.2 \mathrm{~V}^{16,23}$, which prevents the formation of molecular junction at their anti-resonance states. Comparing the conductance variation of 2,4-TP-SMe (20 times) and 2,4-TP-SAc (27 times) in the potential range from $-0.2 \mathrm{~V}$ to 
$1.0 \mathrm{~V}$, the reduction of electrode-molecule coupling from covalent $\mathrm{Au}-\mathrm{S}$ bond to coordinating $\mathrm{Au}-\mathrm{SMe}$ bond do not provide more effective conductance tuning of the single-molecule junctions upon varying electrode potentials.
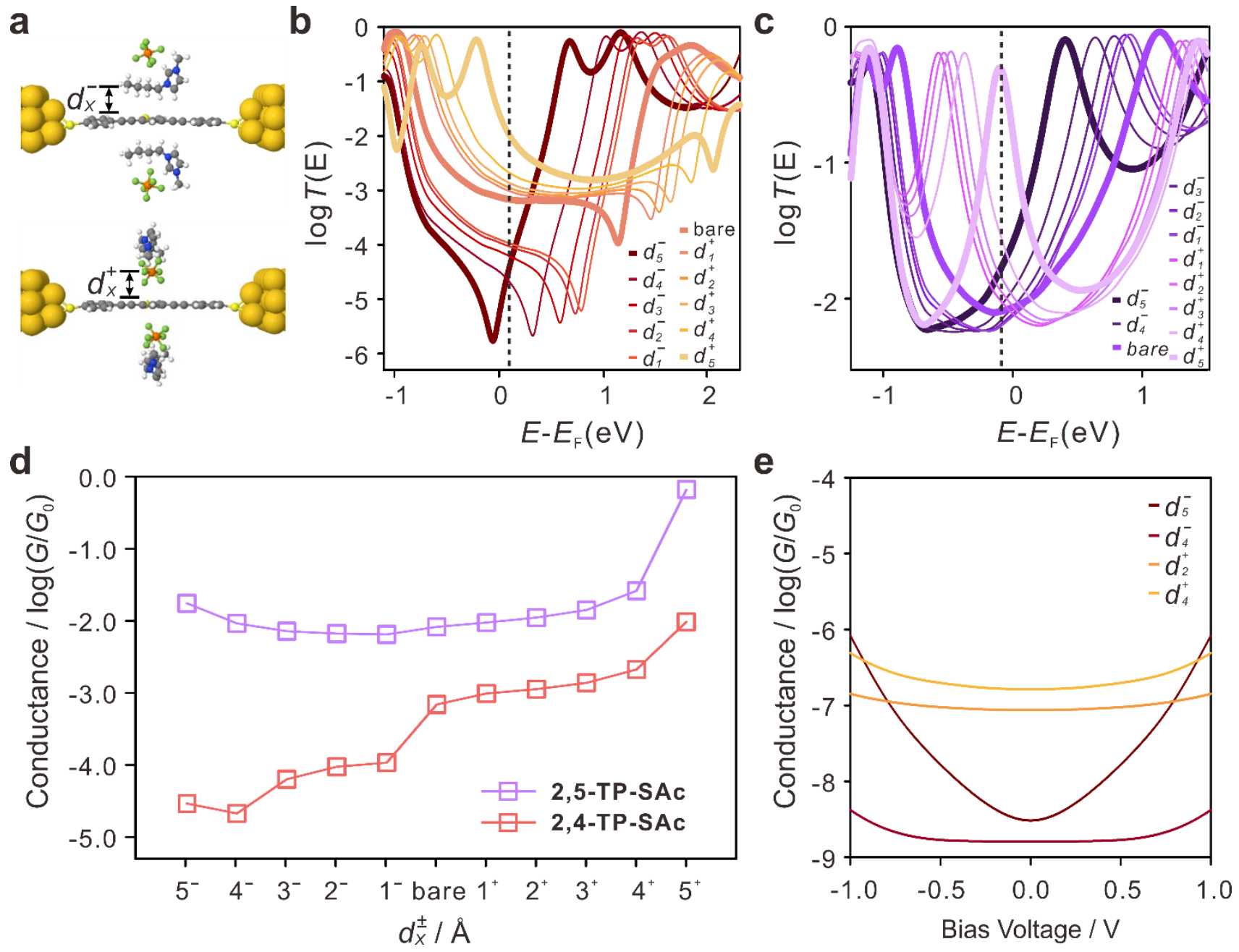

Fig. 4 | Transport properties of thiophene core molecular junctions in the absence and presence of HMIPF6. a, Structure of 2,4-TP-SAc molecular junctions under negative potential (up) and positive potential (down). b, Transmission coefficients of 2,4-TP-SAc in presence of negative and positive charges with different positions. c, Transmission coefficients of 2,5-TP-SAc in presence of negative and positive charges with different positions. d, Comparison of 2,4-TP-SAc and 2,5-TP-SAc conductance versus the position of $\mathrm{HMIPF}_{6}$. The Fermi energy (black dashed line) lies around the middle of the highest occupied molecular orbital and lowest unoccupied molecular orbital (HOMOLUMO) gap $\left(E_{\mathrm{F}}=0.1 \mathrm{eV}\right)$ of bare junction for 2,4-TP-SAc and $-0.08 \mathrm{eV}$ for 2,5-TP-SAc. e, Calculated conductance versus bias voltage of 2,4-TP-SAc for different positions of $\mathrm{HMIPF}_{6}$. 
In order to understand the effect of the electrochemical gating on the single-molecule junctions, we performed quantum transport calculations ${ }^{28,29}$ of the junctions formed by thiophene cores with 2,4 and 2,5 connectivities to the Au electrodes via thiol or -SMe as anchoring group, both in the absence and presence of $\mathrm{HMIPF}_{6}$ (see Methods and Supplementary Fig. 9). The transmission coefficient of the bare junction in the absence of $\mathrm{HMIPF}_{6}$ shows a clear DQI feature for 2,4-TP-Sac, while 2,5-TP-SAc shows no DQI features (Fig. 4b, c). When an electric field is applied, depending on the orientation of $\mathrm{HMIPF}_{6}$, the transmission coefficient shifts to the left or right. $d_{\bar{x}}^{ \pm}$in Fig. 4 denotes the positions of $\mathrm{HMIPF}_{6}$ from the backbone and the potential orientation (" $d_{x}^{-}$" represents the position of HMIPF 6 from the molecular backbone at negative potentials and " $d_{x}^{+}$" represents the $\mathrm{HMIPF}_{6}$ position of from the molecular backbone at positive potentials. " $\mathrm{x}$ " ranges from 1 to 5 correspond to the decreased distance of $\mathrm{HMIPF}_{6}$ from the molecular backbone, and the detailed distances were shown in Supplementary Fig. 9). When the HMI moiety is closer to the back bone ( $d_{x}^{-}$in Fig. 4$), T(E)$ shifts to the left, whereas it shifts to the right when $\mathrm{PF}_{6}$ gets closer to back bone $\left(d_{x}^{+}\right.$in Fig. 4$)$. It is found that HOMO-LUMO gap also shrinks owing to the electric field. In addition, the anti-resonance dip associated with DQI in 2,4-TP-SAc is moved by the position of $\mathrm{HMIPF}_{6}$. Clearly, if the Fermi energy lies close to the anti-resonance of $T(E)$ in $\mathbf{2 , 4 - T P - S A c}$, a large variation of the conductance is predicted (red curves in Fig. 4b). Conversely, when $\mathrm{HMIPF}_{6}$ approaches the backbone from the $\mathrm{PF}_{6}$ side, the dip of $T(E)$ moves to the right, away from the DFT predicted Fermi energy (black dashed line) and the HOMO moves closer, which leads to the near-resonance charge transport (yellow curves in Fig. 4b). In 2,5-TP-SAc (Fig. 4c, d), $T(E)$ does not have a dip and therefore a smaller variation is predicted unless the Fermi energy happens to be in the tail of resonances. Fig. 4d shows the theoretical variation of the conductance versus the position of $\mathrm{HMIPF}_{6}$ from the back bone for both 2,4-TP-SAc and 2,5TP-SAc. For 2,4-TP-Sac, the conductance initially decreases with a negative field from $d_{1}^{-}$to $d_{4}^{-}$, but then increases from $d_{4}^{-}$to $d_{5}^{-}$, suggesting the presence of an anti-resonance dip from DQI effect 
at $d_{4}^{-}$states, which originates from the crossing of DQI dip at Fermi energy as shown in Fig. 4b. It is also found that the HOMO moves closer at positive potentials, suggesting the presence of nearresonance charge transport, in agreement with the significant conductance increase of 2,4-TP-SAc at positive potentials shown in Fig. 1e. The calculated conductance difference between the anti-resonance $\left(d_{4}^{-}\right.$state) and near resonance ( $d_{5}^{+}$state) is determined to be around two orders of magnitudes, which is quite close to the experimental ratio of $\sim 100$. In comparison, the conductance variation of 2,5-TPSAc is much smaller, which is ascribed to the absence of a DQI feature in the transmission of 2,5-TPSAc. We have also calculated the conductance-voltage traces for 2,4-TP-SAc, which qualitatively agree with the experimentally determined conductance-voltage traces, suggesting the gating towards an anti-resonance leads to more pronounced voltage dependence of conductance at $d_{4}^{-}$state and $d_{5}^{-}$ state. We can also conclude that the most tilted shape of the experimental conductance-voltage traces for 2,4-TP-SAc at $-0.6 \mathrm{~V}\left(d_{5}^{-}\right.$state $)$arises when the Fermi level is located between a resonance and anti-resonance, which leads to the sharpest transmission changes ${ }^{24,26,27}$ (see Supplementary Information S5 for details).

In summary, we employed electrochemical gating for the fine tuning of charge transport properties in single-molecule thiophene junctions with and without DQI using a modified electrochemical MCBJ technique. Benefiting from the high gating efficiency of electrochemical gating and the large potential windows of ionic liquid, this work provides the experimental observation of charge transport at an anti-resonance state arising from DQI at room temperature, which is also supported by DFT simulations of the electrochemical double layer. It is found that the gating of single-molecule thiophene junctions with DQI when the Fermi level is located between a resonance and anti-resonance, shows $\sim 100$ times conductance variation by tuning the electrode potential from $-0.4 \mathrm{~V}$ to $1.3 \mathrm{~V}$ versus the $\mathrm{Ag} / \mathrm{AgCl}$ quasi-reference electrode in ionic liquid, which is more than one order of magnitude higher than that without DQI. The electrochemical tuning of DQI presented in this work provides a 
promising design strategy for tuning of interference in future molecular materials and devices.

Data availability. The data that support the findings of this study are available from the corresponding author upon reasonable request.

\section{References}

1. Guedon, C. M. et al. Observation of quantum interference in molecular charge transport. Nat. Nanotechnol. 7, 305-309 (2012).

2. Lambert, C. J. Basic concepts of quantum interference and electron transport in single-molecule electronics. Chem. Soc. Rev. 44, 875-888 (2015).

3. Garner, M. H. et al. Comprehensive suppression of single-molecule conductance using destructive $\sigma$-interference. Nature 558, 415-419 (2018).

4. Frisenda, R., Janssen, V. A. E. C., Grozema, F. C., van der Zant, H. S. J. \& Renaud, N. Mechanically controlled quantum interference in individual $\pi$-stacked dimers. Nat. Chem. 8, 1099 (2016).

5. Baer, R. \& Neuhauser, D. Phase coherent electronics: A molecular switch based on quantum interference. J. Am. Chem. Soc. 124, 4200-4201 (2002).

6. Hsu, L. Y. \& Rabitz, H. Single-molecule phenyl-acetylene-macrocycle-based optoelectronic switch functioning as a quantum-interference-effect transistor. Phys. Rev. Lett. 109, 186801 (2012).

7. Bergfield, J. P., Solomon, G. C., Stafford, C. A. \& Ratner, M. A. Novel quantum interference effects in transport through molecular radicals. Nano Lett. 11, 2759-2764 (2011).

8. Manrique, D. Z. et al. A quantum circuit rule for interference effects in single-molecule electrical junctions. Nat. Commun. 6, 6389 (2015).

9. Sangtarash, S. et al. Searching the hearts of graphene-like molecules for simplicity, sensitivity, and logic. J. Am. Chem. Soc. 137, 11425-11431 (2015).

10. Liu, X. S. et al. Gating of quantum interference in molecular junctions by heteroatom substitution. Angew. Chem. Int. Ed. 56, 173-176 (2017).

11. Huang, C. et al. Single-molecule detection of dihydroazulene photo-thermal reaction using break junction technique. Nat. Commun. 8, 15436 (2017).

12. Song, H. et al. Observation of molecular orbital gating. Nature 462, 1039-1043 (2009).

13. Perrin, M. L. et al. Large tunable image-charge effects in single-molecule junctions. Nat. Nanotechnol. 8, 282 (2013).

14. Huang, C., Rudnev, A. V., Hong, W. \& Wandlowski, T. Break junction under electrochemical gating: Testbed for singlemolecule electronics. Chem. Soc. Rev. 44, 889-901 (2015).

15. Nichols, R. J. \& Higgins, S. J. Single molecule nanoelectrochemistry in electrical junctions. Acc. Chem. Res. 49, 26402648 (2016).

16. Capozzi, B. et al. Tunable charge transport in single-molecule junctions via electrolytic gating. Nano Lett. 14, 14001404 (2014).

17. Xiang, L. et al. Gate-controlled conductance switching in DNA. Nat. Commun. 8, 14471 (2017).

18. Baghernejad, M. et al. Highly-effective gating of single-molecule junctions: An electrochemical approach. Chem. Commun. 50, 15975-15978 (2014).

19. Brooke, R. J. et al. Single-molecule electrochemical transistor utilizing a nickel-pyridyl spinterface. Nano Lett. $\mathbf{1 5}$, 275-280 (2015)

20. Kay, N. J. et al. Single-molecule electrochemical gating in ionic liquids. J. Am. Chem. Soc. 134, 16817-16826 (2012). 
21. Ruiz, M. P. et al. Bioengineering a single-protein junction. J. Am. Chem. Soc. 139, 15337-15346 (2017).

22. Ting, T.-C. et al. Energy-level alignment for single-molecule conductance of extended metal-atom chains. Angew. Chem. Int. Ed. 54, 15734-15738 (2015).

23. Capozzi, B. et al. Mapping the transmission functions of single-molecule junctions. Nano Lett. 16, 3949-3954 (2016).

24. Hong, W. et al. Single molecular conductance of tolanes: Experimental and theoretical study on the junction evolution dependent on the anchoring group. J. Am. Chem. Soc. 134, 2292-2304 (2012).

25. Li, J., Shen, Y., Zhang, Y. \& Liu, Y. Room-temperature ionic liquids as media to enhance the electrochemical stability of self-assembled monolayers of alkanethiols on gold electrodes. Chem. Commun. 0, 360-362 (2005).

26. Guo, S., Hihath, J., Díez-Pérez, I. \& Tao, N. Measurement and statistical analysis of single-molecule current-voltage characteristics, transition voltage spectroscopy, and tunneling barrier height. J. Am. Chem. Soc. 133, 19189-19197 (2011).

27. Leary, E. et al. Detecting mechanochemical atropisomerization within an STM break junction. J. Am. Chem. Soc. 140, 710-718 (2018).

28. Hatef, S. Theory of electron, phonon and spin transport in nanoscale quantum devices. Nanotechnology 29, 373001 (2018).

29. Ferrer, J. et al. Gollum: A next-generation simulation tool for electron, thermal and spin transport. New J. Phys. 16, 093029 (2014).

Supplementary Information is available in the online version of the paper.

Acknowledgements This research was supported by the National Key R\&D Program of China (2017YFA0204902), National Natural Science Foundation of China (21503179, 21673195, 21703188), the Program for Professor of Special Appointment (Eastern Scholar) at Shanghai Institutions of Higher Learning, Natural Science Foundation of Shanghai (17ZR1447100), Science and Technology Commission of Shanghai Municipality (14DZ2261000), China Postdoctoral Science Foundation (2017M622060), for funding work in Xiamen and supported by EC FP7 ITN 'MOLESCO' project number 606728, UK EPSRC grant EP/N017188/1, EP/M014452/1 for funding instrumentation used in Lancaster. We thank Prof. Zhong-Qun Tian and Prof. Bing-Wei Mao, Xiamen University for the fruitful discussion.

Author Contributions W. H and J. B. originally convinced the idea and designed the experiments. W. H., C. L., and W. C. co-supervised the project. J. B., W. H., A. D., and S. S. wrote the manuscript with input from all authors. J. B., X. L. and X. H. carried out the break junction experiments and analysed the data. Q. Z. and W. C. synthesized and provided the structural characterization of the 
molecules. J. B. and S. L. performed the cyclic voltammetry measurements. Y. T., G. M., J. S. and W. H. built the electrical measurement instrument and wrote the software to control the break junction setup. A. D., S. S., C. L. and H. S. performed the theoretical modelling. All authors conceived the work and discussed the experiments.

Author Information Reprints and permission information is available at www.nature.com/reprints. The authors declare no competing financial interests. Readers are welcome to comment on the online version of the paper. Correspondence and requests for materials should be addressed to $\mathrm{W}$. $\mathrm{H}$. (whong@xmu.edu.cn), C. L. (‥lambert@lancaster.ac.uk), or W. C. (wenbochen@ shiep.edu.cn).

\section{Methods}

\section{$\underline{\text { Synthesis }}$}

The target molecules were prepared by the Sonogashira coupling of 4-ethynylthioanisole and 1(thioacetyl)-4-ethynylbenzene with 2,5-dibromothiophene and 2,4-dibromothiophene, respectively. 4ethynylthioanisole and 1-(thioacetyl)-4-ethynylbenzene were prepared according to published procedures (see Supplementary Information S1)

\section{$\underline{\text { Chip fabrication }}$}

Materials for chip fabrication are prepared as follow: $\mathrm{Ag} / \mathrm{AgCl}$ wire was fabricated by electrochemically oxidizing Ag wire (0.1 mm diameter, 99.99\%, Jiaming, Beijing) at a constant potential of $1.5 \mathrm{~V}$ in the electrolyte of $\left(37 \% \mathrm{HCl}: \mathrm{H}_{2} \mathrm{O}=1: 1, \mathrm{v} / \mathrm{v}\right)$. The coated Au tip was prepared by etching $\mathrm{Au}$ wire $(0.15 \mathrm{~mm}$ diameter, $99.99 \%$, Jiaming, Beijing $)$ in a solution of $(37 \% \mathrm{HCl}:$ ethanol $=$ $1: 1, \mathrm{v} / \mathrm{v}$ ) at a potential of $1.2 \mathrm{~V}$ and then coated with hot melt adhesive (Ace Hardware Corp., USA). In our study, the Au beads of $0.35-0.50 \mathrm{~mm}$ in diameter were annealed by butane flame. The electrodes were fixed onto a sheet of spring steel $(30 \mathrm{~mm} \times 10 \mathrm{~mm}$ with $0.2 \mathrm{~mm}$ thickness $)$ using two drops of epoxy (Stycast 2850 FT with catalyst 9). 
Fabrication process of ECTB chip was as follow: the spring steel sheets $(30 \mathrm{~mm} \times 10 \mathrm{~mm} \times 0.2 \mathrm{~mm})$ were cleaned by acetone and Milli-Q water, and dried by nitrogen flow. The coated Au tip wire and Pt wire $(0.1 \mathrm{~mm}$ diameter, $99.95 \%)$ were fixed on the sheet with one drop of epoxy (mixture of STYCAST 2850 FT epoxy resin and 3.5 wt.\% Catalyst 9). After complete polymerization at room temperature, the $\mathrm{Au}$ bead and $\mathrm{Ag} / \mathrm{AgCl}$ wire were fixed on the other side of the sheet by one drop of epoxy. The initial distance between coated Au tip and Au bead was adjusted to be less than $50 \mu \mathrm{m}$ under optical microscope (SAIKEDIGITAL, SK2700U). Before the experiment, the as-prepared chip was rinsed by isopropanol and dried by natural volatilization.

\section{Electrochemical measurements}

The CV measurements at ambient conditions were employed through our laboratory-built bipotentiostat, while an Autolab electrochemical workstation (Eco Chemie, Netherlands) was used when measured in glovebox filled with ultra-pure argon.

The stability of the $\mathrm{Ag} / \mathrm{AgCl}$ quasi-reference electrode was tested by open-circuit potential measurements using a two-electrode system ${ }^{30}$. The working electrode and the reference electrode were the $\mathrm{Ag} / \mathrm{AgCl}$ quasi-reference electrode and a commercial saturated calomel electrode ( $\mathrm{SCE}, \mathrm{CH}$ Instruments Inc.), respectively. The two electrodes were set separately ( $\mathrm{Ag} / \mathrm{AgCl}$ quasi-reference electrode was placed in the $\mathrm{HMIPF}_{6}$ ionic liquid, and $\mathrm{SCE}$ was placed in a saturated $\mathrm{KCl}$ aqueous solution) and connected through an agar- $\mathrm{KNO}_{3}-\mathrm{HMIPF}_{6}$ filled salt bridge. The potential signal outputs from the two electrodes were recorded through an Autolab electrochemical workstation (Eco Chemie, Netherlands).

\section{$\underline{\text { Conductance measurements }}$}

Before the experiments, the as-prepared chip was rinsed by isopropanol and dried by natural volatilization. Then the chip was installed with the polytetrafluoroethylene (PTFE) liquid cell on the top fixed by two Al alloy plates (see Supplementary Information S2 for details). The thiophene 
derivatives (see Supplementary Information S1 for synthetic method and characterization) were prepared to be $0.5 \mathrm{mM}$ in a solvent of $\mathrm{HMIPF}_{6}$ (purchased from lolitec ionic Liquids Technologies, Germany). During the single-molecule conductance measurements, $10-15 \mu \mathrm{L}$ molecular solution was added into the PTFE liquid cell.

The electrochemically MCBJ measurements are based on a laboratory-built bipotentiostat with tunable logarithm current-voltage converter ${ }^{31}$. During experiments, the bias voltage between two working electrodes of coated Au tip and Au bead was fixed to be $0.1 \mathrm{~V}$, and the current passing through the working electrodes was measured by a logarithmic $I-V$ converter with a sampling rate of $10 \mathrm{kHz}$ using microcontroller for data acquisition. During the measurements, the ECTB chip was bent down and up with the Al alloy plates, which were driven by a combination of a stepper motor (Zaber NA14B16) and a piezo stack (Thorlab AE0505D18F). The evolution of conductance characteristics was recorded for further analysis during the repeated connecting/breaking process. A statistical analysis was used for the determination of the conductance and stretching displacement distribution of molecular junctions. The construction of $1 \mathrm{D}$ conductance histograms from typical individual traces provides a way to quantify the most probable conductance. In order to reveal the evolution process of singlemolecule junctions, 2D conductance-displacement histograms were constructed. The distance of the electrodes on the ECTB chip were determined from the tunneling current as reported in our previous paper $^{24}$, and the relative displacement distributions were calibrated from the stretching rate determined from pure solvent measurements (see Supplementary Information S4.2 for details). In this case, the tunneling decay constants in pure solvent and molecule containing solution are considered to be the same to simplify the calibration although the tunneling decay constant varied after adding molecules into the solution.

\section{Conductance-electrode potential measurements}

The conductance-electrode potential measurements were carried out in the same environmental 
conditions with the electrochemically-gated conductance measurement. Instead of the fixed potential in the electrochemically-gated conductance measurement, the break junction experiment was started with a preset potential at $-0.2 \mathrm{~V}$. Once the conductance reached the conductance range of molecular plateau (setting $4 \times 10^{-5} \mu \mathrm{A}$ for 2,4-TP-SAc and $8 \times 10^{-4} \mu \mathrm{A}$ for 2,5-TP-SAc), we employed the microcontroller to keep the distance of the two electrodes at a certain value, and started to sweep the gate voltage from -0.2 to $-0.6 \mathrm{~V}$ at $1 \mathrm{~V} / \mathrm{s}$ with fixed bias voltage between the two electrodes. The current were recorded until the junction broke with a sampling rate of $10 \mathrm{kHz}$. Once the junction broke, another break junction process was started to form the single-molecule junction for conductanceelectrode potential measurements. Since the measured current from the forward sweeping represented the total contribution from Faraday current from electrochemical charging and tunneling current from molecular junctions, only the backward sweeping was used for the plotting in the two-dimensional intensity histogram for both molecules. Hundreds of individual curves with mean conductance between $10^{-4}$ to $10^{-6} G_{0}$ are selected for further statistics. The 2D histograms of conductance-electrode potential were constructed with a bin size of $500 \times 500$ in $2 \mathrm{D}$ space.

\section{Current/conductance-voltage measurements}

The current/conductance-voltage measurements were carried out in the same environmental conditions with the electrochemically-gated conductance measurement. Instead of the fixed bias in the electrochemically-gated conductance measurement, the bias between $-1.0 \mathrm{~V}$ and $+1.0 \mathrm{~V}$ continuously was swept at the sweeping rate of $5 \mathrm{~V} / \mathrm{s}$. The conductance at $100 \mathrm{mV}$ as feedback to control the piezo movement for repeating opening and closing process, as reported in our previous paper ${ }^{24,32}$. The stretching rate is reduced to be around $0.1 \mathrm{~nm} / \mathrm{s}$, and several individual traces were collected from each break junction cycles with a sampling rate at $10 \mathrm{kHz}$. The low-bias conductance of the current/conductance-voltage curves are determined from the data within the range from $-100 \mathrm{mV}$ to $100 \mathrm{mV}$. The 2D histograms of conductance versus voltage were constructed with a bin size of 1000 
$\times 1000$ in $2 \mathrm{D}$ space and the bin size of conductance distributions of low-bias conductance is $0.03 \log$ $\left(G / G_{0}\right)$

\section{Theoretical calculations}

Theoretical calculations were performed with a combination of $a b$ initio DFT package SIESTA and the quantum transport code Gollum. Due to electrochemical gating, the concentration of charge double layer formed by positively charged 1-hexyl-3-methylimidazolium (HMI) and negatively charged hexafluorophosphate $\left(\mathrm{PF}_{6}\right)$ varied around the molecular backbone, which also changes the local electric field applied to the backbone. Depending on the electrode potentials, either positively or negatively charged ends of $\mathrm{HMIPF}_{6}$ are oriented towards the backbone. We accounted for the variation in the effective electric field by moving the $\mathrm{HMIPF}_{6}$ with fixed orientation towards the backbone. Fig. 3a shows two different configurations of 2,4-TP-SAc molecular junctions in the presence of $\mathrm{HMIPF}_{6}$, in which negative or positive region of $\mathrm{HMIPF}_{6}$ is closer to the backbone to simulate the electrochemical gating at positive and negative potentials. We calculated the material specific mean field Hamiltonian ${ }^{33}$ of each structure (see Supplementary Fig. 9) and then combined it with the quantum transport code Gollum ${ }^{29}$ to calculate transmission coefficient $T(E)$ of electrons with energy $E$ passing from one electrode to the other. The electrical conductance $G$ is then calculated from transmission coefficient $G=G_{0} T\left(E_{\mathrm{F}}\right)$ where $E_{\mathrm{F}}$ is the Fermi energy of the electrodes. (see Supplementary Information S5 for details). The conductance for different bias voltages can also be calculated from $G=I / V$ where $I(V)=\int d E T(E)\left(f\left(E-\frac{e V}{2}\right)-f\left(E+\frac{e V}{2}\right)\right)$ and $f$ is the Fermi Dirac distribution function $^{28}$. In order to calculate the current/conductance-voltage characteristic of junctions in the linear response regime, zero-bias transmission coefficient $T(E)$ combined with Landauer formula was employed ${ }^{28}$.

Data availability. The data that support the findings of this study are available from the corresponding 
author upon reasonable request.

\section{References for methods}

30. Shitanda, I., Kiryu, H. \& Itagaki, M. Improvement in the long-term stability of screen-printed planar type solid-state ag/agcl reference electrode by introducing poly(dimethylsiloxane) liquid junction. Electrochim. Acta 58, 528-531 (2011).

31. Meszaros, G., Li, C., Pobelov, I. \& Wandlowski, T. Current measurements in a wide dynamic range - applications in electrochemical nanotechnology. Nanotechnology 18, 424004 (2007).

32. Hong, W. et al. An MCBJ case study: The influence of $\pi$-conjugation on the single-molecule conductance at a solid/liquid interface. Beilstein J. Nanotechnol. 2, 699-713 (2011).

33. José, M. S. et al. The SIESTA method for $a b$ initio order- N materials simulation. J. Phys-Condens. Mat. 14, $2745-$ 2779 (2002). 